\title{
Chlorophyll extraction from Egyptian Luffa leaf
}

\section{Cang H. Mai*, Nguyen H. Nguyen, Giao T. Huynh, \& Y T. N. Le}

Department of Chemical Engineering, Nong Lam University, Ho Chi Minh city, Vietnam

\author{
ARTICLE INFO \\ Research Paper \\ Received: July 19, 2018 \\ Revised: December 22, 2018 \\ Accepted: February 28, 2019 \\ Keywords \\ Chlorophyll \\ Egyptian Luffa \\ Egyptian Luffa leaf \\ ${ }^{*}$ Corresponding author \\ Mai Huynh Cang \\ Email: maihuynhcang@hcmuaf.edu.vn
}

\section{ABSTRACT}

This study examined factors affecting on chlorophyll extraction from Egyptian Luffa leaf for using as food colorant. Optimal conditions for chlorophyll extraction were ethanol $96 \%$ for 97 minutes at $49^{\circ} \mathrm{C}$ and extraction speed at $123 \mathrm{rpm}$. The quality of extracts was investigated for microorganisms, heavy metals andantioxidant activity by using the DPPH (2-2-diphenyl-1DPPH ) method. The free radical scavenging activities of extract presented by the $\mathrm{IC}_{50}$ value was $261,7 \mu \mathrm{g} / \mathrm{mL}$.

Cited as: Mai, C. H., Nguyen, N. H., Huynh, G. T., \& Le, Y. T. N. (2019). Chlorophyll extraction from Egyptian Luffa leaf. The Journal of Agriculture and Development 18(4), 99-107. 


\title{
Nghiên cứu tách chiết Chlorophyll từ lá mướp (Egyptian Luffa)
}

\author{
Mai Huỳnh Cang*, Nguyễn Hồng Nguyên, Huỳnh Thị Giao \& Lê Thị Như Ý \\ Bộ Môn Công Nghệ Hóa Học, Trường Đại Học Nông Lâm TP.HCM, TP. Hồ Chí Minh
}

\author{
THÔNG TIN BÀI BÁO \\ Bài báo khoa học \\ Ngày nhận: 19/07/2018 \\ Ngày chỉnh sửa: 22/12/2018 \\ Ngày chấp nhận: 28/02/2019 \\ Từ khóa \\ Chlorophyll \\ Egyptian Luffa \\ Lá mướp \\ *Tác giả liên hệ \\ Mai Huỳnh Cang \\ Email: maihuynhcang@hcmuaf.edu.vn
}

\section{TÓM TẮT}

Nghiên cứu này khảo sát các yếu tố ảnh hưởng đến quá trình chiết chlorophyll từ lá mướp nhằm ứng dụng tạo màu trong thực phẩm. Điều kiện tối ưu để tách chiết chlorophyll trong lá mướp là chiết ngâm dầm với ethanol $96 \%$, thời gian chiết là 97 phút, nhiệt độ chiết là $49^{\circ} \mathrm{C}$, tốc độ khuấy là 123 vòng/phút. Chế phẩm màu được xác định chỉ tiêu vi sinh vật, kim loại nặng và khả năng kháng oxi hóa bằng phương pháp $\mathrm{DPPH}$ (2-2-diphenyl-1-picrylhydrazyl). Khả năng bắt gốc tự do của chlorophyll thể hiện qua giá trị $\mathrm{IC}_{50}$ là $261,7 \mu \mathrm{g} / \mathrm{mL}$.

\section{1. Đặt Vấn Đề}

Cây mướp hương (Egyptian Luffa) là một loại cây thảo dạng dây leo sống ở vùng nhiệt đới và cận nhiệt đới, lá đơn dạng mọc cách, phiến lá hình trái xoan, đáy hình tim, mép lá có răng cưa (Nguyen, 2016). Lá mướp khi già được sử dụng để tạo các thực phẩm có màu xanh tương tự như lá dứa, ngoài ra lá mướp còn được sử dụng như một loại thảo dược do chúng có chứa các chất có hoạt tính sinh học, đặc biệt là khả năng kháng khuẩn và kháng oxi hóa. Theo Nguyen (2016), lá mướp có khả năng kháng các loại vi khuẩn như Bacillus Subtilis, Escherichia Coli, Staphylococcus Aureus, Samonela Typhi do có chứa saponin, alkaloid và glycoside. Thành phần hóa lý trong lá mướp tươi trình bày Bảng 1 (Nguyen, 2016). Chất diệp lục (chlorophyll) là một sắc tố màu xanh lá cây được tìm thấy trong hầu hết tất cả các thực vật, tảo và vi khuẩn Cyanobacteria. Chất diệp lục là một sắc tố màu xanh lá cây bao gồm vòng tetrapyrrole với một ion magiê ở trung tâm, nó có một đầu kỵ nước dài là chuỗi phytol trong cấu trúc phân tử (Rajalakshmi \& Banu,
2015). Có hai loại chất diệp lục chính là chloropyll $\mathrm{a}$ và chlorophyll $\mathrm{b}$ (Hosikian \& ctv., 2010). Sự khác biệt giữa hai chất diệp lục này là một nhóm methyl trong chlorophyll a được thay thế bởi một nhóm formyl trong chlorolophyll b (Rajalakshmi \& Banu, 2015).

Chlorophyll a là sắc tố chính trong thực vật, chúng chuyển đổi năng lượng ánh sáng thành năng lượng hóa học thông qua quá trình quang hợp (Costache \& ctv., 2012). Vì chlorophyll là các sắc tố xanh có chứa một vòng porphyrin xung quanh các electron tự do nên chúng có thể nhận hoặc cho electron dễ dàng. Vì vậy, sau khi hấp thụ năng lượng của ánh sáng, chlorophyll sẽ cung cấp các electron năng lượng tới một bộ tế bào để bắt đầu quá trình quang hợp (Ridwan \& ctv., 2017). Quang hợp là một quá trình sử dụng năng lượng ánh sáng cùng với nước và carbon điôxit để tạo ra oxy và carbohydrate cung cấp cho sự sống trên trái đất (Hosikian \& ctv., 2010).

Tính chất lý học quan trọng nhất là chlorophyll có khả năng hấp thụ năng lượng áng sáng chọn lọc. Quang phổ hấp thụ cực đại của chlorophyll vùng tia xanh $(\lambda: 430-460 \mathrm{~nm})$ và vùng ánh 
Bảng 1. Thành phần hóa lý trong lá mướp tươi ${ }^{1}$

\begin{tabular}{cccccccc}
\hline \multicolumn{7}{c}{ Trong 100 g lá mướp tươi } \\
\hline Nước & Năng lượng & Chất đạm & Chất béo & Chất xơ & Tro & Calcium & Phosphate \\
$(\mathrm{g})$ & $(\mathrm{kcal})$ & $(\mathrm{g})$ & $(\mathrm{g})$ & $(\mathrm{g})$ & $(\mathrm{g})$ & $(\mathrm{mg})$ & $(\mathrm{mg})$ \\
94 & 14 & 1,6 & 0,1 & 2,7 & 1,6 & 330 & 33 \\
\hline
\end{tabular}

Nguồn: Nguyen (2016).

sáng đỏ ( $\lambda$ : 620 - $700 \mathrm{~nm})$. Chlorophyll dễ bị biến đổi thành pheophytin (có màu olive) trong môi trường acid và nhiệt độ cao do hydro thay vào vị trí nhân Mg (Ngo, 2012).

Hiện nay, chlorophyll được nghiên cứu và sử dụng rộng rãi trong nhiều lĩnh vực như: chất màu tự nhiên trong thực phẩm (Putra \& ctv., 2017); tổng hợp trong ngành hóa học và vật lý cho các ứng dụng khác nhau là điện tử, photophysics, quang điện, điện hóa (Rajalakshmi \& Banu, 2015); cung cấp các lợi ích sức khỏe như chống đột biến và chống oxy hóa có vai trò tích cực trong việc phòng - tránh bệnh ưng thư $(\mathrm{Pu}-$ tra \& ctv., 2017). Đặc biệt, với bản chất không độc hại của chlorophyll, tính chất kháng khuẩn và khử mùi nên chlorophyll là một sản phẩm chủ chốt trong điều trị nhiễm trùng miệng, loét mô, khối u, ung thư (Hosikian \& ctv., 2010).

Mục tiêu của nghiên cứu là khảo sát các yếu tố ảnh hưởng đến quá trình tách chiết chlorophyll từ lá mướp nhằm mục đích tạo chế phẩm màu tự nhiên dùng trong chế biến biến thực phẩm.

\section{Vật Liệu và Phương Pháp Nghiên Cứu}

\subsection{Nguyên liệu}

Nguyên liệu chính được sử dụng là lá mướp hương (Egyptian Luffa) trồng tại Long An. Lá tươi (từ 4 đến 5 tháng, kích thước lá dài 8 - 16 $\mathrm{cm}$, rộng $7-20 \mathrm{~cm}$ ) rửa sạch, sấy khô tại $53^{0} \mathrm{C}$, xay nhỏ (kích thước $0,5 \mathrm{~mm}<\mathrm{d}<1 \mathrm{~mm}$ ), bảo quản trong túi zip ở nhiệt độ phòng (có silicagel hút ẩm).

Hóa chất sử dụng: Ethanol 99\%, KI 99,9\%, I2 99,9\%, 1- $\alpha$ naphthol 99,9\%, $\mathrm{H}_{2} \mathrm{SO}_{4} 98 \%$, acid acetic $99 \%, \mathrm{FeCl}_{3} 99,9 \%, \mathrm{NaOH} 20 \%$, $\mathrm{HCl} 36,5 \%$, chloroform $99 \%$ có nguồn gốc từ Trung Quốc, được mua tại Công ty TNHH Hóa Chất Bách Khoa, TP. Hồ Chí Minh.

Thiết bị sử dụng: Máy sấy khay (Bộ môn Công nghệ hóa, Đại học Nông Lâm TP. Hồ Chí Minh), máy cô quay chân không Stuart RE 300B (Mỹ), máy quang phổ UV-VIS Genesys 20 (Mỹ), cân điện tử 3 số $(\mathrm{Mỹ})$, bể điều nhiệt Memmert WNB (Đức).

\subsection{Phương pháp nghiên cứu}

2.2.1. Khảo sát thành phần hóa lý của nguyên liệu lá mướp

Xác định chỉ tiêu hóa lý: độ ẩm (sấy, cân không đổi), hàm lượng tro tổng (TCVN-5253-90), đo màu (máy so màu Lab, Chroma meter CR-400).

2.2.2. Định tính thành phần hóa học nguyên liệu (Chakraboty \& ctv., 2017)

Alkaloid: $0,5 \mathrm{~mL}$ mẫu $+4-5$ giọt dung dịch $\left(2 \mathrm{~g} \mathrm{KI}+1,27 \mathrm{~g} \mathrm{I}_{2}+100 \mathrm{~mL} \mathrm{H} \mathrm{H}_{2} \mathrm{O}\right)$. Dung dịch phản ứng có màu đỏ nâu.

Carbohydrates: Hòa tan 3,75 g 1- $\alpha$ naphthol + $25 \mathrm{~mL}$ ethanol $99 \%+2 \mathrm{~mL}$ mẫu $+2 \mathrm{~mL} \mathrm{H} \mathrm{SO}_{4}$ vào ống nghiệm sau đó trộn dung dịch, để yên 2-3 phút. Dung dịch phản ứng tách thành 2 màu đỏ và tím.

Glycoside: $5 \mathrm{~mL}$ mẫu $+2 \mathrm{~mL}$ acid acetic +1 giọt $\mathrm{FeCl}_{3}$ sau đó thêm từ từ $\mathrm{H}_{2} \mathrm{SO}_{4}$ đậm đăc. Dung dịch phản ứng có vùng màu nâu bề mặt.

Flavanoid: $2 \mathrm{~mL}$ mẫu + vài giọt $\mathrm{NaOH} 20 \%$. Dung dịch phản ứng có màu vàng và bị mất màu khi thêm $\mathrm{HCl}$ loãng.

Phenol: 1 phần mẫu + dung dịch $\mathrm{FeCl}_{3} 5 \%$. Dung dịch phản ứng có màu xanh đậm hoặc đen.

Tannins: $2 \mathrm{~mL}$ mẫu $+1 \mathrm{~mL} \mathrm{HCl} \mathrm{1 \% .} \mathrm{Sau} \mathrm{phản}$ ứng có kết tủa đỏ.

Saponin: $2 \mathrm{~mL}$ mẫu $+6 \mathrm{~mL} \mathrm{H}_{2} \mathrm{O}$ lắc mạnh dung dịch. Xuất hiện dịch khoảng 5 phút.

Terpenoids: $1 \mathrm{~mL}$ Chloroform $+2 \mathrm{~mL}$ mẫu + vài giọt $\mathrm{H}_{2} \mathrm{SO}_{4}$ đậm đặc. Sau phản ứng có kết tủa nâu hơi đỏ.

\subsubsection{Khảo sát quá trình tách chiết chlorophyll trong lá mướp}

Chiết chlorophyll trong lá mướp bằng phương pháp ngâm dầm, nguyên liệu khô có kích thước 
từ $0,5 \mathrm{~mm}<\mathrm{d}<1 \mathrm{~mm}$. Tỉ lệ nguyên liệu/dung môi là $1 / 30$. Khảo sát hàm lượng chiết chlorophyll khi thay đổi các yếu tố độ ẩm nguyên liệu (sau thời gian sấy 3 giờ, 5 giờ, 7 giờ và 8 giờ), nồng độ dung môi (ethanol 99,5, 96, 80 và $70 \%, \mathrm{v} / \mathrm{v}$ ), nhiệt độ chiết (nhiệt độ phòng, 40 và $50^{\circ} \mathrm{C}$ ), tốc độ khuấy $(50,100,300$ và 500 vòng/phút) và thời gian khuấy (30,60, 90 và 120 phút).

Dùng phương pháp trắc quang UV - VIS để xác định hàm lượng chlorophyll trong dịch chiết. Sau khi cô quay chân không để đuổi dung môi, chất khô sẽ được đem pha loãng để tiến hành đo quang. Lấy $2,5 \mathrm{ml}$ dịch chiết pha loãng cho vào cuvet có kích thước $12,5 \times 12,5 \times 45 \mathrm{~mm}$. Hệ số A nếu không vượt quá 2 sẽ được chấp nhận. Hàm lượng cholorophyll được tính theo phương pháp đo quang phổ kế UV-Vis.

\subsubsection{Phương pháp bề mặt đáp ứng (response sur-} face methodology) - kiểu quay tâm

Ba thông số của quá trình chiết: Thời gian khuấy $\left(\mathrm{X}_{1}\right)$, nhiệt độ khuấy $\left(\mathrm{X}_{2}\right)$ và tốc độ khuấy $\left(\mathrm{X}_{3}\right)$. Thí nghiệm được bố trí theo kiểu trục tâm quay (Rotatable Central Composite Design) với 15 thí nghiệm để xác định ảnh hưởng của các yếu tố và tối ưu hóa quá trình chiết chlorophyll a.

Phương trình tổng quát: $\mathrm{Y}=\mathrm{aX}_{1}^{2}+\mathrm{bX}_{2}^{2}+$ $c \mathrm{X}_{3}^{2}+\mathrm{abX} \mathrm{X}_{2}+\mathrm{ac} \mathrm{X}_{1} \mathrm{X}_{3}+\mathrm{bcX}_{2} \mathrm{X}_{3}$.

Trong đó: $\mathrm{a}, \mathrm{b}, \mathrm{c}$ lần lượt là hệ số của các thông số $\mathrm{X}_{1}, \mathrm{X}_{2}, \mathrm{X}_{3}$; Y là hàm lượng chlorophyll.

\subsubsection{Xác định các chỉ tiêu vi $\sinh$ vật, kim loại} nặng của màu chlorophyll

Chế phẩm chlorophyll được đem đánh giá các chỉ tiêu vi sinh vật $(E$. Coli, Salmonella), kim loại nặng $(\mathrm{Pb}, \mathrm{Hg})$, vi sinh vật hiếu khi theo tiêu chuẩn 46/2007 QĐ-BYT.

\subsubsection{Khảo sát khả năng kháng oxi hóa của chloro- phyll theo phương pháp DPPH}

Theo cơ chế bắt gốc tự do sẽ chuyển gốc tự do DPPH (1,1-diphenyl-2-picrylhydrazyl) từ màu tím sang vàng nhạt. Xác định khả năng bắt gốc tự do của chất nghiên cứu bằng phương pháp đo độ hấp thu của mẫu tại bước sóng $\lambda=517 \mathrm{~nm}$. Ascorbic acid được sử dụng làm chất đối chiếu.

Phần trăm bắt gốc tự do DPPH của chất nghiên cứu được tính theo công thức sau:

$$
\mathrm{Q}(\%)=\left(1-\frac{\mathrm{A}-\mathrm{A}_{\mathrm{c}}}{\mathrm{A}_{0}-\mathrm{A}_{\mathrm{c}}}\right) \times 100
$$

Trong đó:

A là độ hấp thu của dung dịch chứa mẫu thử

$\mathrm{A}_{0}$ là độ hấp thu của $\mathrm{DPPH}$ khi không có mẫu

$\mathrm{A}_{\mathrm{c}}$ là độ hấp thu của dung dịch chứa chất đối chiếu

Giá trị $\mathrm{IC}_{50}$ được tính bằng phần mềm GraphPad Prism thông qua đường chuẩn phần trăm ức chế (đường chuẩn được xây dựng từ 6 nồng độ khác nhau).

\subsubsection{Phương pháp bố trí thí nghiệm và xử lí số liệu}

Thí nghiệm được bố trí theo kiểu ngẫu nhiên hoàn toàn, lặp lại 3 lần. Số liệu được tính toán bằng phần mềm thống kê Statgraphics centurion $\mathrm{XV}$ để xác định độ ảnh hưởng của các yếu tố lên hàm lượng chlorophyll ở độ tin cậy 95\%, sự khác biệt giữa các nghiệm thức thông qua bảng LSD. Sau khi thực hiện tất cả các thí nghiệm sơ bộ, tiến hành bố trí thí nghiệm tối ưu trục tâm quay Rotatable Central Composite Design để chọn ra điều kiện chiết chlorophyll phù hợp nhất.

\section{Kết Quả và Thảo Luận}

\section{1. Định tính thành phần hóa học nguyên liệu}

Kết quả trong Bảng 2 cho thấy trong dịch chiết lá mướp có chứa alkaloid, glycoside và saponin. Alkaloid và glycoside là những thành phần tạo vị đắng cho lá mướp, saponin là thành phần dược lý có hoạt tính sinh học giúp tạo nên dược tính cho lá mướp. Hàm lượng tro tổng trong lá mướp nguyên liệu khoảng 2.5\%. Độ ẩm theo cơ sở ướt là $79,9 \%$ (Bảng 3 )

Bảng 2. Định tính thành phần hóa học trong dịch chiết lá mướp

\begin{tabular}{lcc}
\hline Thành phần & Có & Không \\
\hline Alkaloid & $\mathrm{X}$ & $\mathrm{X}$ \\
Carbohydrates & & \\
Glycoside & $\mathrm{X}$ & $\mathrm{X}$ \\
Flavanoid & & $\mathrm{X}$ \\
Phenol & & $\mathrm{X}$ \\
Tannins & $\mathrm{X}$ & $\mathrm{X}$ \\
Saponin & $\mathrm{X}$ \\
Terpenoids & & $\mathrm{X}$ \\
Quinones & & \\
Oxalate & & \\
\hline
\end{tabular}


Bảng 3. Các thành phần khác trong lá mướp tươi

\begin{tabular}{|c|c|c|c|c|}
\hline Thành phần khác & \multicolumn{4}{|c|}{ Hàm lượng } \\
\hline Hàm lượng tro tổng & \multicolumn{4}{|c|}{$2,598 \% \pm 0.55$} \\
\hline Độ ẩm (wb) & \multicolumn{4}{|c|}{$79,9 \% \pm 2.85$} \\
\hline \multirow{2}{*}{ Đo màu } & Mặt đậm & $\mathrm{L}^{*}=37,297$ & $a^{*}=-10,077$ & $\mathrm{~b}^{*}=15,137$ \\
\hline & Mặt nhạt & $\mathrm{L}^{*}=48,467$ & $a^{*}=-11,077$ & $\mathrm{~b}^{*}=18,580$ \\
\hline
\end{tabular}

Bảng 4. Hàm lượng chlorophyll trong lá mướp so với các nguyên liệu giàu chlorophyll phổ biến khác

\begin{tabular}{lc}
\hline Nguyên liệu & Lượng chlorophyll (mg/g) \\
\hline Lá mướp & 4,31 \\
Lá dứa (Le, 2000) & 4,91 \\
Súp lơ xanh (Garcia \& ctv., 2000) & 2,95 \\
Tảo Spirulina (Duong \& ctv., 2010) & 1,0 \\
Rau bina (Le, 2014) & 6,98 \\
\hline
\end{tabular}

Bảng 5. Kết quả độ ẩm lá mướp sau khi sấy

\begin{tabular}{cccccc}
\hline Thời gian sấy (giờ) & 3 & 5 & 7 & 8 & 9 \\
Độ ẩm $(\%)$ & $35,08 \pm 1,05$ & $30,92 \pm 1,22$ & $24,23 \pm 0,95$ & $20,12 \pm 1,06$ & $20,01 \pm 0,85$ \\
\hline
\end{tabular}

Bảng 4 cho thấy, hàm lượng chlorophyll trong lá mướp là khoảng $4,31 \mathrm{mg} / \mathrm{g}$, tương đương với lá dứa (là loại lá có hàm lượng chlorophyll cao phổ biến, thường được dùng để tạo màu trong chế biến thực phẩm). Hiện nay xu hướng chiết chlorophyll từ tảo biển khá phổ biến do lượng chlorophyll chiết được cao đồng thời không qua giai đoạn tinh sạch phức tạp, tuy nhiên việc dùng lá mướp chiết chlorophyll có thể tận dụng nguồn nguyên liệu rẻ tiền sãn có giúp nâng cao hiệu quả kinh tế của cây mướp hương.

Hình 1 \& Bảng 5 cho thấy hàm lượng chlorophyll thay đổi theo thời gian sấy và độ ẩm nguyên liệu. Thời gian sấy 8h (độ ẩm khoảng 20,12\%), hàm lượng chlorophyll bắt đầu giảm. Kết quả phân tích ANOVA cho thấy thời gian sấy ảnh hưởng có ý nghĩa lên hàm lượng chlorophyll ở độ tin cậy 95\%. Hàm lượng chlorophyll cao nhất ở thời gian sấy 7 giờ và khác biệt có ý nghĩa so với các thời gian sấy khác $(P<0,05)$. Chọn thời gian sấy 7 giờ cho các thí nghiệm tiếp theo. Nếu tiếp tục sấy lên 8 giờ thì hàm lượng chlorophyll a bắt đầu giảm vì chlorophyll dễ bị phân hủy ở nhiệt độ trên $50^{\circ} \mathrm{C}$ khi sấy trong thời gian dài và hàm lượng acid có trong dịch bào của lá kết hợp với nhiệt độ cao làm chlorophyll chuyển hóa thành pheophytin (có màu xanh olive). Ngược lại, với thời gian sấy thấp hơn 7 giờ thì độ ẩm của nguyên liệu còn cao làm giảm hiệu suất tách chlorophyll; đồng thời, độ ẩm cao còn là nguyên nhân góp phần đẩy nhanh quá trình oxy hóa chlorophyll trong nguyên liệu (Putra \& ctv., 2017).

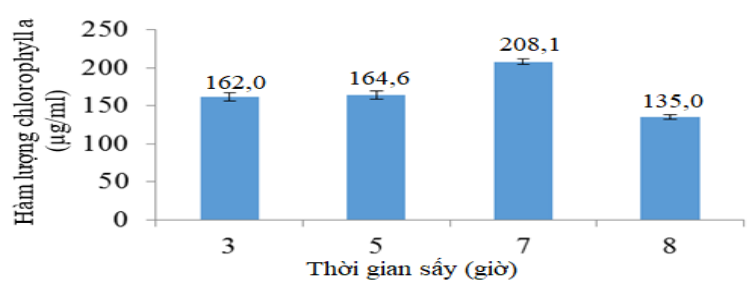

Hình 1. Ảnh hưởng của thời gian sấy đến hàm lượng chlorophyll a.

\section{2. Ảnh hưởng của nồng độ dung môi tới hàm lượng chlorophyll thu được}

Hình 2 cho thấy hàm lượng chlorophyll thay đổi theo nồng độ dung môi và ở nồng độ ethanol $96 \%$ thì hàm lượng chlorophyll bắt đầu giảm. Kết quả phân tích ANOVA cho thấy ảnh hưởng của nồng độ dung môi lên hàm lượng chlorophyll là có ý nghĩa ở độ tin cậy $95 \%(P<0,05)$, hàm lượng chlorophyll ở nồng độ 99,5\% là cao nhất nhưng do khác biệt không ý nghĩa với nồng độ $96 \%$ ở độ tin cậy $95 \%$ nên chọn ethanol $96 \%$ làm dung môi cho các thí nghiệm sau. Nồng độ ethanol càng cao, lượng nước trong dung môi ít, độ phân cực của dung môi giảm nên chlorophyll dễ dàng ra khỏi nguyên liệu. Ethanol $70 \%$ có hàm lượng nước khá 
cao (chiếm $42 \%$ thể tích) nên mẫu bị oxi hóa do oxi trong nước sẽ phản ứng với chlorophyll khi tiếp xúc trong thời gian dài sẽ làm mẫu chiết có màu ngả sang vàng nên không tiến hành xác định hàm lượng chlorophyll.

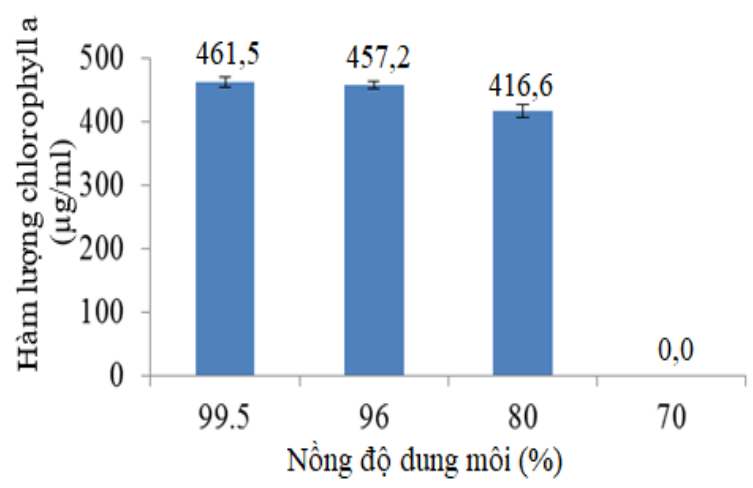

Hình 2. Ảnh hưởng của nồng độ dung môi đến hàm lượng chlorophyll a.

\section{3. Ảnh hưởng của nhiệt độ chiết tới hàm lượng chlorophyll a thu được}

Hình 3 cho thấy hàm lượng chlorophyll thay đổi theo nhiệt độ và tăng ở nhiệt độ $50^{\circ} \mathrm{C}$. Kết quả phân tích ANOVA cho thấy ảnh hưởng của nhiệt độ lên hàm lượng chlorophyll là có ý nghĩa ở độ tin cậy $95 \%(P<0,05)$, hàm lượng chlorophyll ở $50^{\circ} \mathrm{C}$ là cao nhất và khác biệt có ý nghĩa so với các nghiệm thức khác. Ở $60^{\circ} \mathrm{C}$ thì chlorophyll bắt đầu chuyển sang màu vàng do chlorophyll chuyển thành pheophytin, nên thí nghiệm chỉ dừng lại ở nhiệt độ $50^{\circ} \mathrm{C}$ để đảm bảo chlorophyll không bị biến đổi. Nhiệt độ tăng, quá trình thẩm thấu dung môi vào thành tế bào và hòa tan chlorophyll vào dung môi diễn ra nhanh hơn, đồng thời quá trình khuếch tán chlorophyll ra khỏi vách tế bào sẽ diễn ra nhanh hơn làm cho lượng chlorophyll thu được càng nhiều.

\section{4. Ảnh hưởng của tốc độ khuấy tới hàm lượng chlorophyll thu được}

Hình 4 cho thấy hàm lượng chlorophyll thay đổi theo tốc độ khuấy và ở tốc độ khuấy 500 vòng/phút hàm lượng chlorophyll giảm. Kết quả phân tích ANOVA cho thấy ảnh hưởng của tốc độ khuấy lên hàm lượng chlorophyll là có ý nghĩa ở độ tin cậy $95 \%(P<0,05)$, hàm lượng chlorophyll ở tốc độ khuấy 300 rpm là cao nhất nhưng

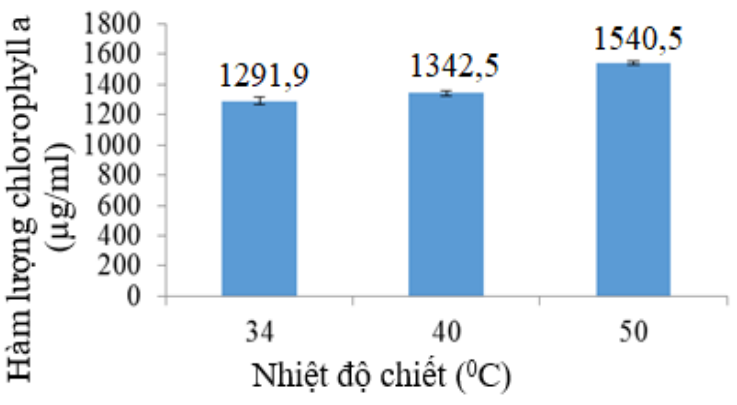

Hình 3. Ảnh hưởng của nhiệt độ chiết đến hàm lượng chlorophyll a.

do khác biệt không có ý nghĩa ở độ tin cậy $95 \%$ với tốc độ khuấy 100 rpm nên chọn 100 rpm cho các thí nghiệm tiếp theo. Tốc độ khuấy càng tăng hàm lượng chlorophyll thu được càng cao do nhiệt độ cao thì các phân tử hạt di chuyển nhanh hơn làm tăng khả năng thẩm thấu qua thành tế bào. Khi tăng tốc độ khuấy lên $500 \mathrm{rpm}$ thì lượng chlorophyll bắt đầu giảm do tốc độ khuấy cao 1 phần chlorophyll sẽ bị oxi hóa, 1 phần do độ nhớt của dịch tăng làm giảm hiệu quả chiết màu.

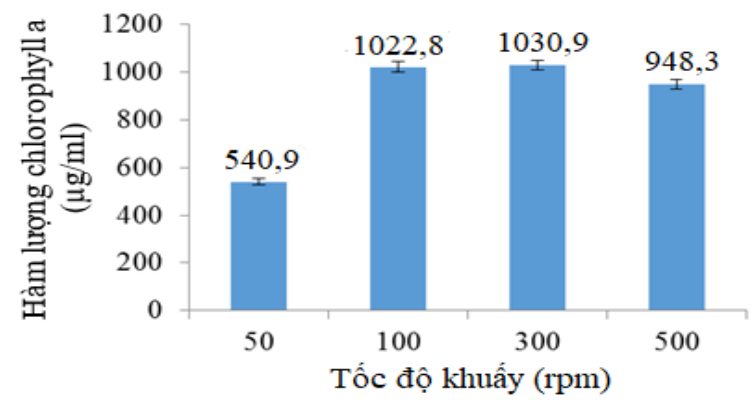

Hình 4. Ảnh hưởng của tốc độ khuấy đến hàm lượng chlorophyll a.

\section{5. Ảnh hưởng của thời gian khuấy tới hàm lượng chlorophyll thu được}

Hình 5 cho thấy hàm lượng chlorophyll thay đổi theo thời gian khuấy và ở thời gian khuây 120 phút thì hàm lượng chlorophyll giảm. Kết quả phân tích ANOVA cho thấy ảnh hưởng của thời gian khuấy lên hàm lượng chlorophyll là có ý nghĩa ở độ tin cậy $95 \%(P<0,05)$, hàm lượng chlorophyll ở 90 phút là cao nhất và khác biệt ý nghĩa ở độ tin cậy 95\%. Thời gian khuấy càng lâu sẽ làm tăng thời gian tiếp xúc giữa nguyên liệu và dung môi nên hàm lượng chlorophyll thu được 
Bảng 6. Sự ảnh hưởng của các yếu tố tối ưu lên hàm lượng chlorophyll

\begin{tabular}{lcccc}
\hline Term & Estimate & Std Error & $\mathrm{t}$ Ratio & Prob > $|\mathrm{t}|$ \\
\hline Intercept & $1.322,191$ & 54,339 & 24,33 & $<, 0001^{*}$ \\
Thời gian khuấy $(60,120)$ & 325,835 & $3.197,027$ & 1,02 & 0,3549 \\
Nhiệt độ khuấy $(45,55)$ & $-485,252$ & $3.197,027$ & $-1,52$ & 0,1895 \\
Tốc độ khuâyy $(0,200)$ & 90,1415 & $3.197,027$ & 2,82 & $0,0371^{*}$ \\
Thời gian khuây* Nhiệt độ khuấy & 983,225 & $3.574,384$ & 0,28 & 0,7943 \\
Thời gian khuấy*Tốc độ khuấy & $-118,745$ & $3.574,384$ & $-0,33$ & 0,7532 \\
Nhiệt độ khuây*Tốc độ khuây & $-28,388$ & $3.574,384$ & $-0,79$ & 0,4631 \\
Thời gian khuấy*Thời gian khuấy & $5.741,772$ & $6.304,622$ & $-0,91$ & 0,4042 \\
Nhiệt độ khuấy*Nhiệt độ khuấy & $1.130,852$ & $6.304,622$ & $-1,79$ & 0,1328 \\
Tốc độ khuây*Tốc độ khuây & 203,7637 & $6.304,622$ & $-3,23$ & $0,0232^{*}$ \\
\hline
\end{tabular}

Bảng 7. Kết quả tối ưu

\begin{tabular}{lcc}
\hline Yếu tố & Giá trị đề xuất & Giá trị làm tròn \\
\hline Thời gian khuấy $(60,120)$ & 97,198794 & 97 \\
Nhiệt độ khuấy $(45,55)$ & 48,834786 & 49 \\
Tốc độ khuấy $(0,200)$ & 123,04329 & 123 \\
Giá trị chlorophyll dự đoán & 1342,1403 & \\
Giá trị chlorophyll thực & $1294,229 \pm 14,305$ & \\
\hline
\end{tabular}

càng cao.

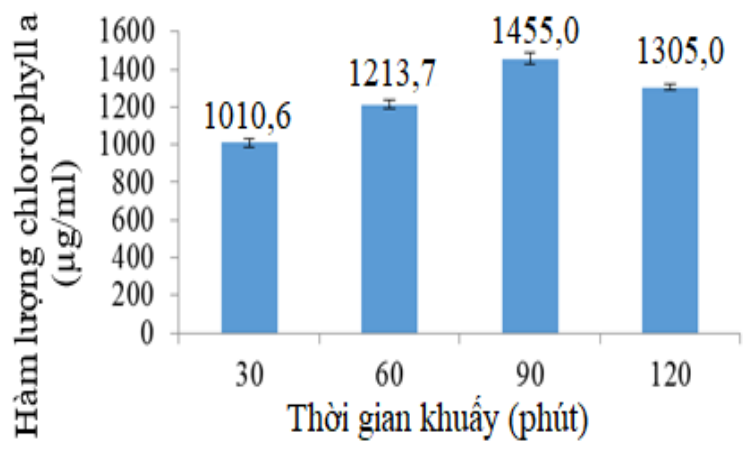

Hình 5. Ảnh hưởng của thời gian khuấy đến hàm lượng chlorophyll a.

\subsection{Tối ưu hóa điều kiện chiết chlorophyll a}

Phương trình hồi quy giữa hiệu suất tách chiết chlorophyll với thời gian khuấy, nhiệt độ khuấy và tốc độ khuấy với hệ số tương quan $\mathrm{R} 2=0,89$ cho thấy chúng có tương quan chặt chẽ (Hình 6).

Bảng 6 cho ta thấy tốc độ khuấy $\left(\mathrm{X}_{3}\right)$ ảnh hưởng có ý nghĩa tới hàm lượng chlorophyll, thời gian khuấy và nhiệt độ khuấy ảnh hưởng không có ý nghĩa. Phương trình đáp ứng hàm lượng chlorophyll như sau:

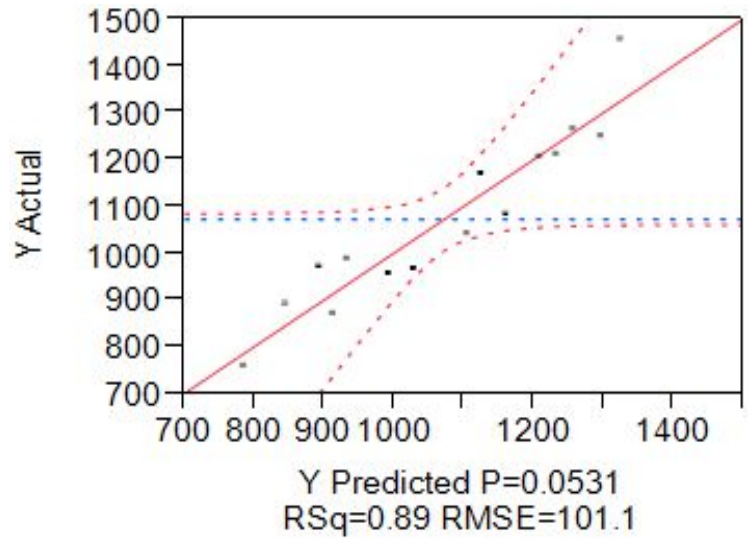

Hình 6. Phương trình hồi quy.

$$
\mathrm{Y}=1.322,191+90,1415 \mathrm{X}_{3}-203,7637 \mathrm{X}_{3}^{2}
$$

Hàm lượng chlorophyll sau khi tiến hành thí nghiệm thực tế có độ chênh lệch 3,57\% (không vượt quá $5 \%$ ) so với hàm lượng chlorophyll dự đoán (Bảng 7). Vì vậy các giá trị thời gian, nhiệt độ và tốc độ khuấy tối ưu để đạt hàm lượng chlorophyll cao nhất lần lượt là 97 phút, $49^{\circ} \mathrm{C}$ và 123 vòng/phút.

\subsection{Khảo sát thành phần hóa lý thành phẩm}

Bảng 8 cho thấy thành phẩm có màu xanh nhạt và tối hơn lá tươi. Quá trình chiết và cô quay loại 
Bảng 8. Kết quả đo màu Lab của thành phẩm

\begin{tabular}{ccccc}
\hline & & $\mathrm{L}^{*}$ & $\mathrm{a}^{*}$ & $\mathrm{~b}^{*}$ \\
\hline Cao chiết chlorophyll & 36,97 & 0,28 & 1,52 \\
\multirow{2}{*}{ Lá tươi } & Mặt đậm & 37,297 & $-10,077$ & 15,137 \\
& Mặt nhạt & 48,467 & $-11,077$ & 18,58 \\
\hline
\end{tabular}

Bảng 9. Định tính thành phần hóa học trong dịch chiết lá mướp

\begin{tabular}{lc}
\hline Thành phần & Kết quả \\
\hline Glycoside & Không \\
Saponin, Alkaloid & Có \\
Độ ẩm & $74,47 \% \pm 2,55$ \\
Hàm lượng cholorophyll $(\mu \mathrm{g} / \mathrm{mL})$ & $1423,069 \pm 22,422$ \\
\hline
\end{tabular}

Bảng 10. Kết quả đo hàm lượng vi sinh vật và kim loại nặng

\begin{tabular}{|c|c|c|c|c|c|}
\hline $\mathrm{TT}$ & Chỉ tiêu & Đơn vị & Kết quả & $\begin{array}{l}\text { Phương } \\
\text { pháp thử } \\
\text { nghiệm }\end{array}$ & $\begin{array}{c}\text { Giới } \\
\text { hạn cho } \\
\text { phép }\end{array}$ \\
\hline 1 & Chì (Pb) & $\mathrm{mg} / \mathrm{kg}$ & $\begin{array}{c}\text { Âm tính } \\
(\mathrm{LOD}=0,01)\end{array}$ & $\begin{array}{c}\text { AOAC } \\
999.11\left(^{*}\right)\end{array}$ & $\begin{array}{c}2,0 \\
\mathrm{mg} / \mathrm{kg}\end{array}$ \\
\hline 2 & Thủy ngân (Hg) & $\mathrm{mg} / \mathrm{kg}$ & $\begin{array}{c}\text { Âm tính } \\
(\mathrm{LOD}=0,01)\end{array}$ & $\begin{array}{l}\text { AOAC } \\
971.21\end{array}$ & $\begin{array}{c}0,05 \\
\mathrm{mg} / \mathrm{kg}\end{array}$ \\
\hline 3 & Tổng số vi khuẩn hiếu khí & $\mathrm{CFU} / \mathrm{g}$ & $2,1 \times 10^{4}$ & $\begin{array}{c}\text { ISO } \\
4833-1: 2013\end{array}$ & $10^{4} / \mathrm{g}$ \\
\hline 4 & Escherichia Coli & $\mathrm{CFU} / \mathrm{g}$ & Âm tính & $\begin{array}{c}\text { ISO } \\
16649-2: 2001\end{array}$ & $3 / \mathrm{g}$ \\
\hline 5 & $\begin{array}{l}\text { Tổng số bào tử nấm men, } \\
\text { nấm mốc }\end{array}$ & $\mathrm{CFU} / \mathrm{g}$ & Âm tính & $\begin{array}{c}\text { ISO } \\
21527-2: 2008\end{array}$ & $10^{2} / \mathrm{g}$ \\
\hline 6 & Salmonella & $\mathrm{CFU} / 25 \mathrm{~g}$ & $\begin{array}{c}\text { Âm tính } \\
(\mathrm{LOD}=0,01)\end{array}$ & $\begin{array}{c}\text { ISO } \\
6579-1: 2017\end{array}$ & $\begin{array}{l}\text { Không } \\
\text { có }\end{array}$ \\
\hline
\end{tabular}

dung môi làm cho chlorophyll bị oxy hóa và biến đổi một phần thành pheophytin nên thành phẩm sẽ có màu vàng chiếm ưu thế hơn màu xanh.

Bảng 9 cho thấy sau quá quá trình cô quay thì trong thành phẩm chỉ còn lại saponin và alkaloid, glycoside mất đi trong quá trình cô quay dựa trên phương pháp định tính màu (Chakraboty \& ctv., 2017). Độ ẩm thành phẩm xác định bằng phương pháp sấy ở nhiệt độ sấy là $53^{0} \mathrm{C}$, cân khối lượng không đổi và giá trị độ ẩm là $74,47 \% \pm 2,55$. Hàm lượng chlorophyll trong thành phẩm xác định bằng phương pháp UV-VIS, nhận thấy hàm lượng chlorophyll là $1423,069 \pm 22,422 \mu \mathrm{g} / \mathrm{mL}$.

Bảng 10 cho thấy trong thành phẩm không phát hiện $\mathrm{Hg}, \mathrm{Pb}$, E.Coli, Salmonella, nấm men, nấm mốc. Tổng số vi khuẩn hiếu khí phát hiện nằm trong giới hạn cho phép theo $\mathrm{QĐ}$ 46/2007 BYT.

\subsection{Xác định khả năng kháng oxi hóa của mẫu theo phương pháp DPPH}

Mẫu Chlorophyll : $\mathrm{IC}_{50}=261,7 \pm 2,108 \mu \mathrm{g} / \mathrm{ml}$; Vitamin $\mathrm{C}: \mathrm{IC}_{50}=16,37 \pm 1,458 \mu \mathrm{g} / \mathrm{mL}$

$\mathrm{IC}_{50}$ là nồng độ của dịch chiết khử được $50 \%$ gốc tự do DPPH ở điều kiện xác định. Giá trị IC50 càng thấp thì hoạt tính khử gốc tự do DPPH càng cao. Khả năng kháng oxi hóa của sản phẩm chlorophyll $\left(\mathrm{IC}_{50}=261,7 \mu \mathrm{g} / \mathrm{mL}=0,262\right.$ $\mathrm{mg} / \mathrm{mL}$ ) thấp hơn gần 16 lần so với vitamin $\mathrm{C}$ $\left(\mathrm{IC}_{50}=0,0164 \mathrm{mg} / \mathrm{mL}\right)$, cao hơn so với lá chùm ngây $\left(\mathrm{IC}_{50}=0,537 \mathrm{mg} / \mathrm{mL}\right.$ ) (Phan \& Nguyen, 2016). Biểu đồ phần trăm bắt gốc tự do DPPH theo nồng độ chlorophyll thể hiện ở Hình 7 . 


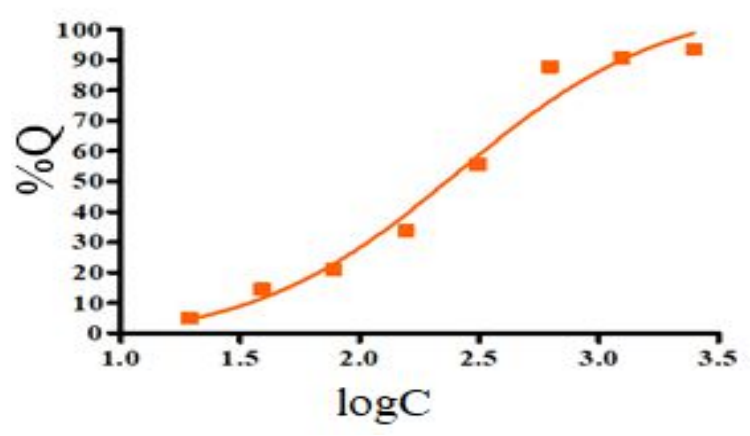

Hình 7. Biểu đồ phần trăm bắt gốc tự do DPPH theo nồng độ chlorophyll.

\section{Kết Luận}

Nghiên cứu đã tiến hành khảo sát các yếu tố ảnh hưởng đến hiệu quả chiết chlorophyll từ lá mướp bao gồm: ngâm chiết bằng ethanol $96 \%$, tốc độ khuấy là 123 vòng/phút, thời gian chiết là 97 phút, nhiệt độ chiết ở $49^{\circ} \mathrm{C}$ là phù hợp nhất. Màu xanh chlorophyll thành phẩm có độ ẩm khoảng $74,47 \%$. Các nghiên cứu tiếp theo cần làm giảm độ ẩm của cao chlorophyll và theo dõi chất lượng sản phẩm theo thời gian bảo quản. Kết quả đánh giá các chỉ tiêu vi sinh vật và kim loại nặng ta nhận thấy chế phẩm màu xanh chlorophyll đạt các chỉ tiêu vi sinh vật và kim loại nặng $(\mathrm{Pb}$, $\mathrm{Hg}$ ) theo quyết định của Bộ Y tế 46/2007 QĐ - BYT. Ngoài ra nghiên cứu cũng khảo sát khả năng kháng oxi hóa của màu xanh chlorophyll từ lá mướp với giá trị $\mathrm{IC}_{50}=261,7 \mu \mathrm{g} / \mathrm{mL}$

\section{Tài Liệu Tham Khảo (References)}

Chakraboty, S., Sahoo, S., Anjana, B., \& Dixit, S. (2017). Studies on antimicrobial activity , phytochemical screening tests, biochemical evaluation of clitorea ternatea linn. Plant extracts 5(10), 197-208.
Costache, A. M., Campeanu, G., \& Neata, G. (2012). Studies concerning the extraction of chlorophyll and total carotenoids from vegetables. Romanian Biotechnological Letters 17, 7702-7708.

Duong, L. T., Le, H. T., \& Vu, T. T. (2010). Functional food - sustainable health, Ha Noi, Vietnam: Science and Technics Publishing House.

Garcia, C., Martinez., T., \& Lopez A. (2000). Measurement of chlorophyll contents in broccoli using spectrophotometer. Journal of Science and Food Agriculture 45(2), 456-460.

Hosikian, A., Lim, S., Halim, R., \& Danquah, M. K. (2010). Chlorophyll extraction from microalgae: A Review on the process engineering aspects. International Journal of Chemical Engineering 39, 32-44.

Le, H. M. (2014). Canned food technology. (Unpublished master's thesis). University of Science, Ha Noi, Vietnam.

Le, N. T. V. (2000). Procedures for collection of Chlorophyll from pandan leaves. (Unpublished master's thesis). HUTECH University, Ho Chi Minh City, Vietnam.

Nguyen, V. T. (2016). Herbal garden: luffa aegyptiaca. Ho Chi Minh City, Vietnam: Tre Publishing House.

Phan, T. T. B., \& Nguyen, M. T. D. (2006). Study of antioxidant activity on leaves and stem of Moringa oleifera. Can Tho University Journal of Science 3, 179-184.

Putra, M. D., Darmawan, A., Wahdini, I., \& Abasaeed, E. A. (2017). Extraction of chlorophyll from pandan leaves using ethanol and mass transfer study. Joural of the Serbian Chemical Society 82, 921-931.

Rajalakshmi, K., \& Banu, N. (2015). Extraction and estimation of chlorophyll from medicinal plants. International Journal of Science and Research 4, 209-212.

Ridwan, A. M., Noor E., Rusli S. M., \& Akhiruddin M. (2017). Introductory Study towards the extraction of chlorophyll pigment from Sargassum. International Journal of Sciences: Basic and Applied Research 35, 211-221. 